\title{
Design of Sentiment Analysis System using Polarity Classification Technique
}

\author{
Rajeshwar Rao Kodipaka \\ Asst.Prof, Dept of CSE, \\ MREC-Sec-bad, TS
}

\author{
Sanjeeva Polepaka \\ Associate.Prof, Dept of CSE, \\ MREC-Sec-bad, TS
}

\author{
Md. Rafeeq \\ Associate.Prof, Dept of CSE, \\ CMRTC-Kandlakoya-Hyd, TS
}

\begin{abstract}
Twitter is a medium that we can use for communication. All posted tweets we can store in one location and create archive. Archive contains new and old tweets. Now we can start the analyzation on archive tweets that's we can design effective sentiment analysis system. This paper main aim is to determine parts of speech opinion words using polarity classification technique and support vector machine learning algorithm. Surveys of methods are used in various levels of sentiment analysis. It does analyze the tweets information in limited levels of content only. Now in this paper we design new sentiment analysis tool using polarity classification technique. Polarity classification techniques discover top 20emoticons, learning different classes of words and other features information. These techniques perform in depth tweets analysis. It does provide better analysis results compare to previous methods.
\end{abstract}

\section{Keywords}

Sentiment analysis, opinion mining, text documents, support vector machine classifier, polarity classification technique.

\section{INTRODUCTION}

Twitter enables all organizations for communication directly with each other. It's possible to tap the global real time communication important events very easy using twitter analysis. It is used to find out opinions from twitter micro blogging tweets. Many numbers of researchers have done good extensive work presently and previously on this topic environment. Analysis starts on the basis of tweets. Present approaches are missing contexts for some number of conclusions.

In this paper using polarity classification technique analyzes the tweets and fulfills the missing context features. Here we fulfill the features like part of speech opinion words of information, frequent occurrences opinions words discovery with the help of support vector machine classifier. It gives more conclusions compare to all previous approaches and more useful also for any real applications decision making.

\section{RELATED WORK}

Sentiment analysis is also known as Opinion Mining. Sentimental analysis major aims to determine the attitude of writer, judgment and communication based on text documents. Sentiment analysis major task is classify text documents, sentences, aspect based level. Express opinions in documents, sentences, aspect based level information are positive, negative or neutral [1].

Twitter data contains different topics information related to different domains. Classify the sentiment documents information using probabilistic model. Probabilistic model is one of the supervised learning algorithm. Each topic related how many documents are available it's not possible to recognize or predictable. First choose the class labels and classify each and every topic documents separately [1] [2]. This is we can call as a document level sentiment classification.

In each topic sentiment documents again possible to classify or learning the sentiment sentences, aspect level process, opinion words also. The above steps are possible to implement on news, blogs and other categories [2] [3].

In twitter first collect the different categories of data using hash tags input. Hash tags works like class labels, but here there is [3] [4] no sentiment labels information. Supervised learning is not sufficient for extraction of sentiment analysis information. Here we should use the unsupervised learning [4] [2]. Using unsupervised learning identify the sentiment words measurement in documents. Sentiment words are categorized into positive and negative contexts.

Sentiment words are huge. Reduces the sentiment words information and improve the classification result. Classification improves using co-occurrence technique. Highly occurred features we can display as a output [4] [5] using unsupervised clustering. All high occurred features are not semantic or meaningful.

On co-occurrence words information applies correlation technique finally recognizes or predicts the relationship sentiment words content. This correlation sentiment words procedure is best prediction procedure compare to above all procedures [2] [3] [5].

Again in twitter it's possible to predict the similar opinions of information. Identify the social relationship users from total twitter data. Social relationship users it's possible to display using visualization concept. Finally it's possible to display temporal events relationship information also as a final result. Consider the temporal events relationship and possible to view of sentiment words trends information effectively. These trends changes dynamically [4] [5] [6].

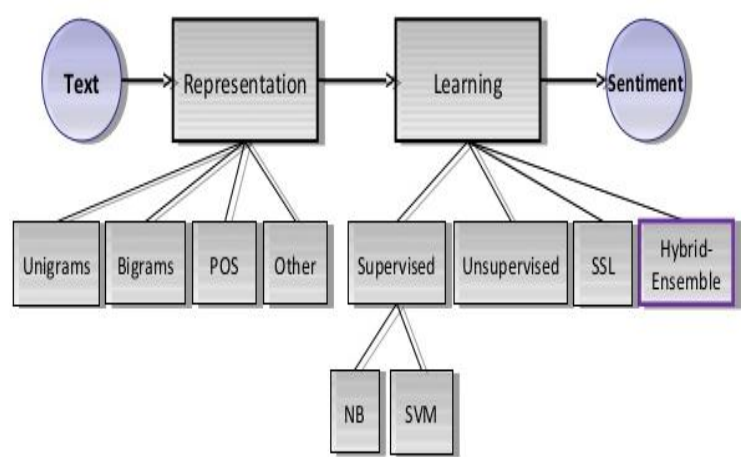

Fig 1: Sentiment Analysis Result with different data mining techniques 


\section{PROBLEM STATEMENT}

Face book and Twitter are major resources for sentiment analysis. Nowadays numbers of people are increases for posting the opinions in social media environment. Result is vast amount of unstructured data is available. Manual sentiment analysis is not feasible which generated data is currently. Here in this paper we can propose automated sentiment analysis techniques. Automated sentiment analysis techniques are extracting different characteristics of information. Here we focus on tweet level polarity classification process. Hence it is one of the interesting research topics.

\section{PROPOSED METHODOLOGY}

After posting before analysis of raw tweets we can apply preprocessing operation. Using preprocessing operation removes the unnecessary content and change the format of context information. Next we use the tokeniser operations for dividing the content based on parts of speech. According to parts of speech classify the sentences of information. Classify the sentences depends on hash tags or tokens. Different hash tags are different parts of speech content purpose in our implementation process.

Here we design the different rules for sentiment analysis. Those rules are emoticon, support vector machines with ngrams, Sentic computing and Linguistic rules etc.

\subsection{Emoticon Rules}

Emoticons are ASCII art. They are formed through creative letters, numbers and symbols. Most of the people represent the facial features information. Here normally for plain text messages we can add the emotional flavor. Next here for normal text messages again we can add the smile automatically those messages are converted to happiness and surprise messages.

First choose the twitter data source and collect lakhs or millions of tweets. In millions of tweets we can perform the analyzation operation and we discovered 20 usage patterns information.

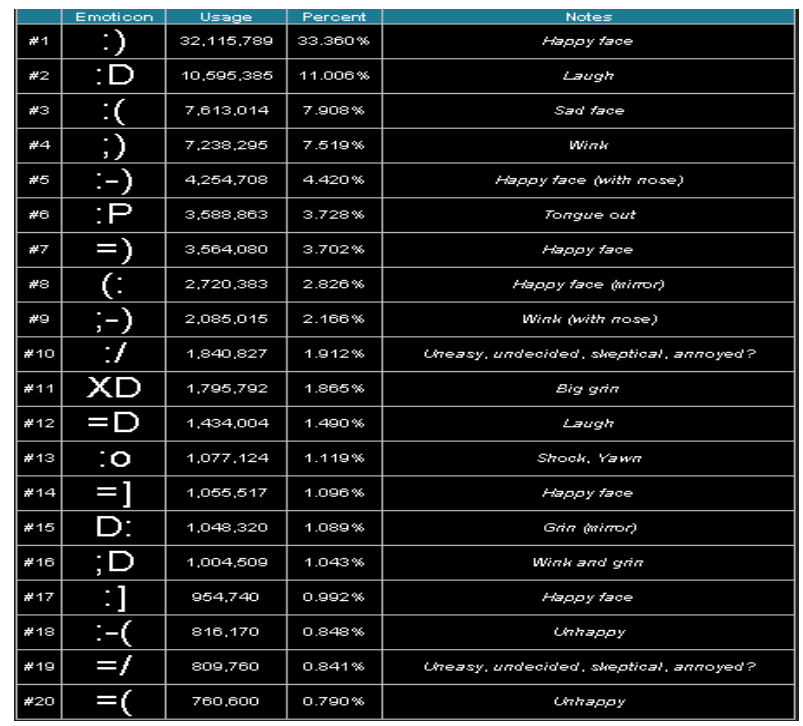

Fig2: Top 20 Emoticons

In Above diagrams top-20 emoticons we displayed here in our implementation. These emoticons are occurred frequently in number of tweets messages.

\subsection{N-Grams Technique}

In posted tweets observe the features and store features into feature vector. All required features are available in sequence or contiguous or not we can check with n-grams concept. Afterwards using TF-IDF identifies and calculates the frequency count. Consider the frequency count generate weighted features information. Weighted features information controls the number of dimensions. In all frequent words we can categorize based on parts of speech. Consider the different number of parts of speech tags information and categorize the words into noun, adverb, verb etc.

\subsection{Linguistic Rules}

After collection of training tweets information next we can apply support vector machine classifier. It will separate the features of information $\mathrm{n}$ different classes. Each and every class contains some data points of information content. Each and every class of words again categorize into two classes. Those two classes are positive and negative words. Calculate the polarity value like positive and negative content. Polarity value is nothing but decision score.

\section{EXPERIMENTS AND RESULTS}

We evaluate our proposed system on available real time datasets. Every and every year tweets we can collect separately and create dataset. Every year dataset contains different categories tweets are available. Those categories are positive, negative and neutral tweets. Here first remove neutral tweets from total number of tweets information.

Evaluate two datasets and calculates efficiently different performance metrics parameters information. Those parameters are precision, recall and f-measure.

Table1: Performance Metrics

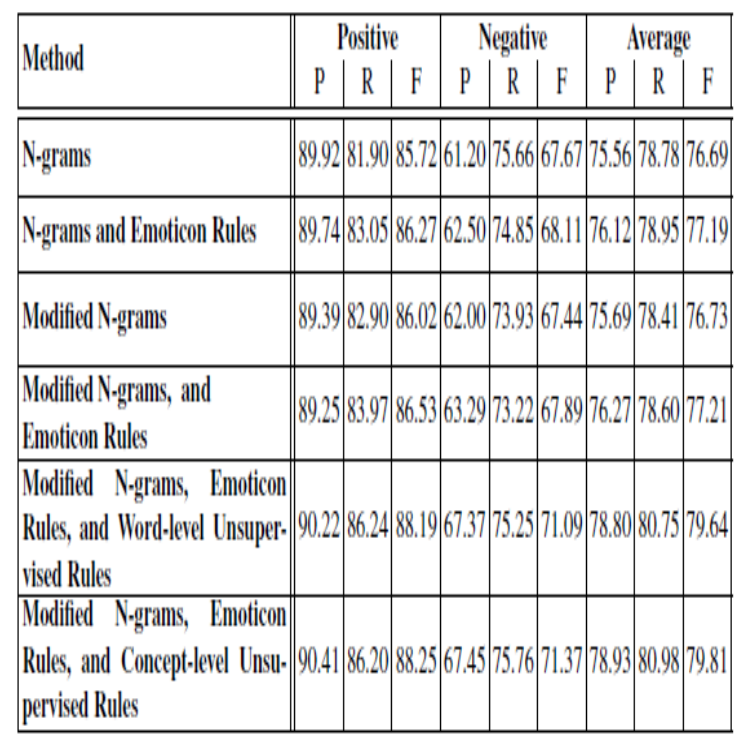

\section{CONCLUSION AND FUTURE WORK}

In this paper new twitter sentiment analysis system apply rules and discover more useful text. Useful text contains meaningful features. Those features are discovered using linguistic content and sentic computing rules. Features are like emoticon symbols and n-grams content information. These meaningful features are support for decision making in all real time applications.

In future we plan to improve the performance using other unsupervised classifiers. We plan to develop some more rules 
for efficient text predictions and multi model sentiment analysis.

\section{REFERENCES}

[1] B. J. Jansen, M. Zhang, K. Sobel, and A. Chowdury, "Twitter power: Tweets as electronic word of mouth," J. Am. Soc. Inform.Sci. Technol., vol. 60, no. 11, pp. 2169-2188, 2009.

[2] B. J. Jansen, M. Zhang, K. Sobel, and A. Chowdury, "Micro-blogging as online word of mouth branding," in Proc. Extended Abstr. Human Factors Comput. Syst., 2009, pp. 3859-3864.

[3] J. Bollen, H. Mao, and X. Zeng, "Twitter mood predicts the stock market," J. Comput. Sci., vol. 2, no. 1, pp. 1-8, 2011.

[4] A. Tumasjan, T. O. Sprenger, P. G. Sandner, and I. M. Welpe, "Predicting elections with twitter: What 140 characters reveal about political sentiment," in Proc. 4th Int. AAAI Conf. Weblogs Soc. Media, 2010, vol. 10, pp. $178-185$.

[5] L. T. Nguyen, P. Wu, W. Chan, W. Peng, and Y. Zhang, "Predicting collective sentiment dynamics from timeseries social media," in Proc. 1st Int. Workshop Issues Sentiment Discovery Opinion Mining, 2012, p. 6.

[6] M. Thelwall, K. Buckley, and G. Paltoglou, "Sentiment in twitter events," J. Am. Soc. Inform. Sci. Technol., vol. 62, no. 2, pp. 406-418, 2011.

[7] A. Agarwal, B. Xie, I. Vovsha, O. Rambow, and R. Passonneau, "Sentiment analysis of twitter data," in Proc. Workshop Lang. Soc. Media, 2011, pp. 30-38.

[8] B. Liu, "Sentiment analysis and opinion mining," Synthesis Lect. Human Lang. Technol., vol. 5, no. 1, pp. 1-167, 2012.

[9] C. Tan, L. Lee, J. Tang, L. Jiang, M. Zhou, and P. Li, "User-level sentiment analysis incorporating social networks," in Proc. $17^{\text {th }}$ ACM SIGKDD Int. Conf. Knowl. Discovery Data Mining, 2011, pp. 1397-1405.

[10] J. Blitzer, M. Dredze, and F. Pereira, "Biographies, bollywood, boom-boxes and blenders: Domain adaptation for sentiment classification," in Proc. 45th Annu. Meeting Assoc. Comput. Linguistics, 2007, vol. 7, pp. $440-447$.
[11] F. Li, S. J. Pan, O. Jin, Q. Yang, and X. Zhu, "Crossdomain coextraction of sentiment and topic lexicons," in Proc. 50th Annu. Meeting Assoc. Comput. Linguistics: Long Papers, 2012, pp. 410-419.

[12] S. J. Pan, X. Ni, J.-T. Sun, Q. Yang, and Z. Chen, "Cross-domain sentiment classification via spectral feature alignment," in Proc. 19th Int. Conf. World Wide Web, 2010, pp. 751-760.

[13] I. Ounis, C. Macdonald, J. Lin, and I. Soboroff, "Overview of the trec-2011 microblog track," in Proc. 20th Text Retrieval Conf., 2011, http://trec.nist.gov/pubs/trec20/t20.proceedings.html

[14] I. Soboroff, I. Ounis, J. Lin, and I. Soboroff, "Overview of the trec- 2012 microblog track," in Proc. 21st Text REtrieval Conf., 2012

[15] A. Go, R. Bhayani, and L. Huang, "Twitter sentiment classification using distant supervision," CS224N Project Report, Computer Science Department, Stanford, USA pp. 1-12, 2009.

[16] S. Li, C.-R. Huang, G. Zhou, and S. Y. M. Lee, "Employing personal/ impersonal views in supervised and semi-supervised sentiment classification," in Proc. 48th Annu. Meeting Assoc. Comput. Linguistics, 2010, pp. 414-423.

\section{AUTHOR PROFILE}

Rajeshwarrao. Kodipaka (ISTE,CSI Life Member), working as Asst.Prof in CSE, MREC since 1 year, Having 7 years teaching experience and interested domain is Data mining, cloud computing, Computer Networks.

Sanjeeva Polepaka is working as associate professor in Malla Reddy Engineering college (Autonomous) Hyderabad ,TS State. He completed B.Tech(CSE) from Andhra University ,M Tech(CSE) from ANU Guntur Perusing Ph D from JNTU Hyderabad. He has 12 years of teaching experience in various engineering colleges. He is life member of CSI and ISTE .His area of interest includes Image processing mobile, grid computing and computer network.

Md. Rafeeq Doing external $\mathrm{PhD}$ in JNTUHHyd,Telangana,India. I would like thank to my guide Dr. Sunil Kumar, and co-guide Dr. Subhash Chandra for constant support for Understanding the subject. 